\section{Neuronal circuits for fear and anxiety — the missing link}

\section{Richard Apps and Piergiorgio Strata}

The recent Review by Tovote et al. (Neuronal circuits for fear and anxiety. Nat. Rev. Neurosci. 16, 317-331 (2015)) ${ }^{1}$ provides a valuable summary of our current understanding from animal studies of the importance of distributed brain networks in fear and anxiety. Given the key role of limbic structures (such as the amygdala, the periaqueductal grey (PAG) and the hippocampus) and closely interlinked cerebral cortical areas (such as the prefrontal cortex), the Review focuses on how these CNS structures drive emotional behaviours, including freezing in response to fearful stimuli. Tovote et al. indicate that this list of structures is not exhaustive. Nonetheless, we would argue that the cerebellum is an important omission.

The cerebellum contains more than $80 \%$ of all neurons in the human brain ${ }^{2}$ and uses its extraordinary computational power to control most, if not all, aspects of behaviour. There is a substantial body of evidence that points to the cerebellum as a crucial component of the neural matrix that subserves emotionally related behaviours (for reviews, see REFS 3,4). This evidence includes consistent findings from human imaging studies of increases in blood oxygen level-dependent signals or metabolic activity within the cerebellum in response to painful or threatening stimuli ${ }^{5}$ and even during mental recall of personally charged episodes ${ }^{6}$. Anatomical and physiological mapping studies have shown that extensive interconnections exist between the cerebellum and important elements of the emotional behaviour network, including the $\mathrm{PAG}^{7}$, the amygdala ${ }^{8}$, the hippocampus ${ }^{8,9}$ and the prefrontal cortex ${ }^{10}$.
Stimulation of the midline vermal region of the cerebellum, or its output, the fastigial nucleus, can elicit various complex patterns of defence-like behaviour, such as sham rage $^{11}$. Moreover, both autonomic ${ }^{12}$ and fear-related conditioning ${ }^{13}$ have been shown to require the integrity of the cerebellar vermis.

In particular, Sacchetti et al..$^{13}$ have shown in rats that rostral parts of the cerebellar vermis (lobules V and VI) are important sites of plasticity related to consolidation of conditioned fear memory (FIG. 1a). Similarly, lesion studies have shown that a more caudal region of the cerebellar vermis (lobule VIII), which has strong physiological connections with the PAG, is essential for the expression of both conditioned and unconditioned freezing ${ }^{7}$ (FIG. 1b,c). A substantial body of evidence therefore indicates that the cerebellum, and particularly its vermal compartment, is crucially involved in both the memory and the expression of emotional behaviour. Accordingly, the cerebellum should be included in the distributed network of brain regions that are associated with fear (FIG. 2). Other studies have shown that the cerebellum is involved in a range of additional

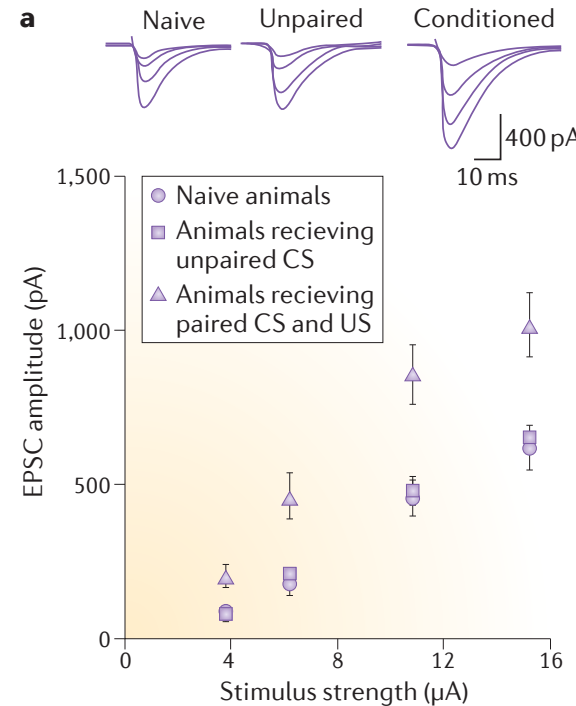

Figure 1 | The cerebellum and fear. a |A long lasting increase in excitatory transmission between parallel fibres and Purkinje cells occurs after fear learning in rats. Stimulation of parallel fibres at increasing strength results in an increased amplitude of the excitatory postsynaptic current (EPSC) evoked in Purkinje cells in vermal lobule $V$ and lobule VI 24 hours after the training. In comparison with naive animals (circles; $n=17$ ) or animals that received an unpaired conditioned stimulus (CS; squares; $n=23$ ), those that received the CS and an unconditioned stimulus (US) in a paired manner exhibited increases in EPSC amplitude, resulting in conditioned fear behaviour (triangles; $n=17$ ). $\mathbf{b}$ | Neurotoxin-induced lesions of the cerebellar vermis (lobule VIII) reduce fear-induced freezing behaviour in rats.
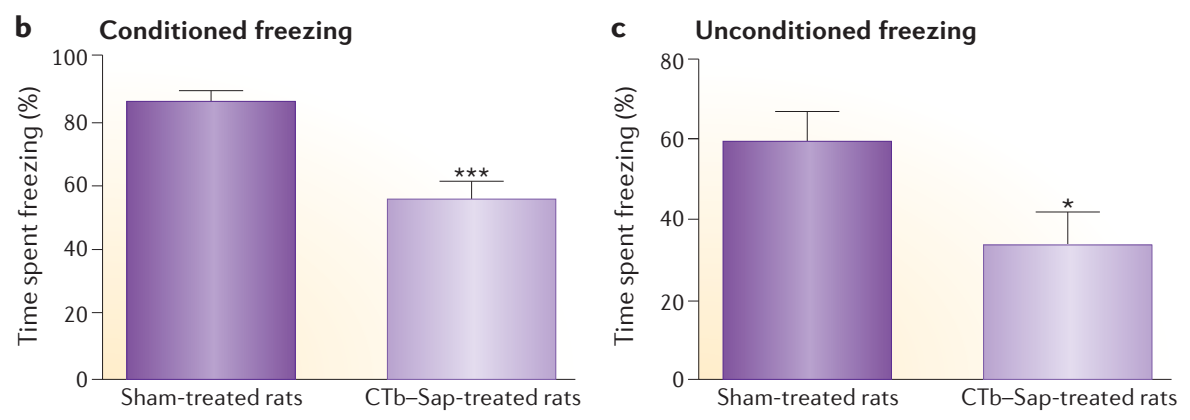


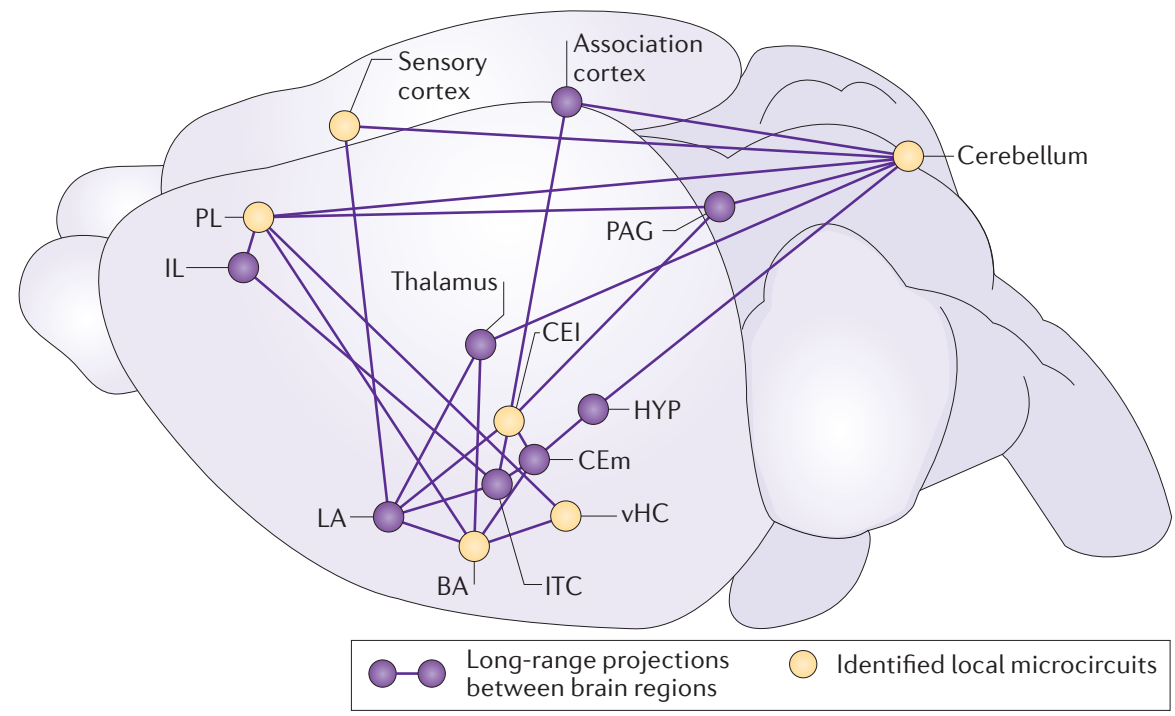

Figure 2 | The fear network. The cerebellum is a key node in the distributed network of brain regions involved in fear-related behaviour. BA, basal amygdala; CEl, lateral central amygdala; CEm, medial central amygdala; HYP, hypothalamus; IL, infralimbic cortex; ITC, intercalated; LA, lateral amygdala; PAG, periaqueductal grey; PL, prelimbic cortex; $\mathrm{vHC}$, ventral hippocampus. Figure adapted from REF. 1, Nature Publishing Group.

cognitive functions ${ }^{14}$. An important question for future investigation is whether the interconnectivity between the cerebellum and the limbic system also contributes to the affective component of emotional behaviours.

Richard Apps is at the School of Physiology, Pharmacology and Neuroscience, Biomedical Sciences Building, University of Bristol, University Walk, Bristol BS8 1TD, UK.
Piergiorgio Strata is at the Department of Neuroscience, University of Turin, Corso Raffaello 30, 10125 Torino, Italy. Correspondence to R.A. e-mail:r.apps@bristol.ac.uk doi: 10.1038/nrn4028

1. Tovote, P., Fadok, J. P. \& Lüthi, A. Neuronal circuits for fear and anxiety. Nat. Rev. Neurosci. 16, 317-331 (2015).

2. Walløe, S., Pakkenberg, B. \& Fabricius, K. Stereological estimation of total cell numbers in the human cerebral and cerebellar cortex Front. Hum. Neurosci. 8, 508 (2014).

3. Watson, T. C. et al. The olivo-cerebellar system and its relationship to survival circuits. Front. Neural Circuits 7, 72 (2013).

4. Strata, P. The emotional cerebellum. Cerebellum http://dx.doi.org/10.1007/s12311-015-0649-9 (2015).

5. Ploghaus, A. et al. Dissociating pain from its anticipation in the human brain. Science $\mathbf{2 8 4}$ 1979-1981 (1999).

6. Damasio, A. R. et al. Subcortical and cortical brain activity during the feeling of self-generated emotions. Nat. Neurosci. 3, 1049-1056 (2000).

7. Koutsikou, S. et al. Neural substrates underlying fear-evoked freezing: the periaqueductal grey-cerebellar link. J. Physiol. 592, 2197-2213 (2014).

8. Heath, R. G. \& Harper, J. W. Ascending projections of the cerebellar fastigial nucleus to the hippocampus, amygdala, and other temporal lobe sites: evoked potential and histological studies in monkeys and cats. Exp. Neurol. 45, 268-287 (1974).

9. Newman, P. P. \& Reza, H. Functional relationships between the hippocampus and the cerebellum: an electrophysiological study of the cat. J. Physiol. 287, 405-426 (1979).

10. Watson, T. C., Becker, N., Apps, R. \& Jones, M. W. Back to front: cerebellar connections and interactions with the prefrontal cortex. Front. Syst. Neurosci. 8, 4 (2014).

11. Zanchetti, A. \& Zoccolini, A. Autonomic hypothalamic outbursts elicited by cerebellar stimulation. J. Neurophysiol. 17, 475-483 (1954).

12. Supple, W. F. \& Leaton, R. N. Cerebellar vermis: essential for classically conditioned bradycardia in the rat. Brain Res. 509, 17-23 (1990).

13. Sacchetti, B. Scelfo, B., Tempia, F. \& Strata, P. Long-term synaptic changes induced in the cerebellar cortex by fear conditioning. Neuron 42, 973-982 (2004).

14. Buckner, R. L. The cerebellum and cognitive function: 25 years of insight from anatomy and

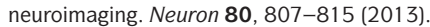

Competing interests statement

The authors declare no competing interests. 